\title{
Prevalence and Correlates of SARS CoV-2 Among a Community-Based Sample Recruited Using Randomized Venue-Based Sampling. Essex County, NJ, 2020
}

\author{
Henry F. Raymond ${ }^{1} \cdot$ Pratik Datta $^{2} \cdot$ Rahul Ukey $^{2} \cdot$ Peng Wang $^{1} \cdot$ Richard J. Martino $^{1} \cdot$ Kristen D. Krause $^{1} \cdot$ \\ Corey Rosmarin-DeStefano ${ }^{3} \cdot$ Abraham Pinter $^{2} \cdot$ Perry N. Halkitis ${ }^{1} \cdot$ Maria L. Gennaro ${ }^{2}$
}

Received: 24 June 2021 / Revised: 29 September 2021 / Accepted: 21 October 2021 / Published online: 8 November 2021

(c) W. Montague Cobb-NMA Health Institute 2021

\begin{abstract}
Disparities in morbidity and mortality related to COVID-19 based on race and ethnicity have been documented in the USA. However, it is unclear if these disparities also exist at the exposure stage. To determine this, studies are needed to document the underlying burden of disease, potential disparities through serologic surveillance. Additionally, such studies can help identify where along the disease spectrum (e.g., exposure, infection, diagnosis, treatment, death) and with regard to the structural factors that necessitate public health and/or clinical interventions. Our objectives in this study were to estimate the true burden of SARS CoV-2 in the community of Essex County, NJ, an early and hard hit area, to determine the correlates of SARS CoV-2 prevalence and to determine if COVID-19 disparities seen by race/ethnicity were also reflected in SARS CoV-2 burden. We utilized venue-based-sampling (VBS) to sample members of the community in Essex County. Participants completed a short electronic survey and provided finger stick blood samples for testing. We sampled 924 residents of Essex County, New Jersey. Testing conducted in this study identified 83 (9.0\%) participants as positive for SARS-CoV-2 antibodies. Importantly, our findings suggest that the true burden of SARS-Cov-2 and the pool of persons potentially spreading the virus are slightly more than six times than that suggested by PCR testing Notably, there were no significant differences in odds of testing positive for SARS CoV-2 antibodies in terms of race/ethnicity where we compared Black and Latinx participants to other race participants. Our study suggests that disparities in COVID-19 outcomes stem from potential upstream issues such as underlying conditions, access to testing, and access to care rather than disparities in exposure to the virus.
\end{abstract}

Keywords SARS CoV-2 $\cdot$ Seroprevalence $\cdot$ Correlates $\cdot$ Disparities

\section{Introduction}

Disparities in the USA based on race and ethnicity with regard to COVID-19 have been documented in terms of morbidity and mortality, but it is unclear if there are similar disparities in exposure to SARS CoV-2 [1-7]. While such patterns parallel other health conditions, the underlying

Henry F. Raymond

hfisher.raymond@rutgers.edu

1 School of Public Health, Rutgers University, Piscataway, NJ, USA

2 Public Health Research Institute, New Jersey Medical School, Rutgers University, Newark, NJ, USA

3 North Jersey Community Research Initiative, Newark, NJ, USA psychosocial and structural mechanisms that enable these disparities continue to be elucidated, minority stressors and social inequities are surely the drivers of the disease as noted in recent conceptualizations of COVID-19 and other diseases [8-10].

To this end, studies are needed to document the underlying burden of disease through serologic surveillance. These types of studies are an important complement to case reporting and data on morbidity and mortality. Additionally, seroprevalence studies empower public health practitioners and others to document potential disparities in the true burden and to identify where along the disease spectrum (e.g., exposure, infection, diagnosis, treatment, death) and with regard to the structural factors that necessitate public health and/or clinical interventions.

For example, if the disparities exist at the exposure stage, interventions might be needed to mitigate the conditions 
which facilitate exposure. In contrast, if the disparities are only seen in outcomes that are exacerbated by underlying medical conditions or access to care, further upstream health interventions may be needed. Such initiatives are critically important since some 30\% infected persons are asymptomatic; thus, our understanding of the burden of disease is informed solely by case reporting and may not capture the actual profile of the diseases particularly in hard to reach and marginalized populations [11].

SARS CoV-2 initially manifested in the locally in the Northeastern region of the USA where morbidity and mortality were extremely high in the second quarter of 2020. Essex County, New Jersey, adjacent to New York City, a COVID-19 epicenter, ranks third in New Jersey as of mid-March 2021 for COVID-19 cases $(69,386)$ and deaths $(2,431)$ translating to a morbidity and mortality rate of approximately 8700 per 100,000 and 305 per 100,000 respectively $[12,13]$. By comparison, the rate of the cases is comparable in the USA, although morbidity is lower at the rate of 164 per 100,000 [14]. The extent to which these differences in morbidity are due to temporal factors (i.e., when the pandemic emerged), structural factors or the interaction of both has yet to be delineated.

Essex County is highly diverse along numerous strata with a majority of residents who are Black or Latino $(62.7 \%)$, a high proportion living in poverty $(15.8 \%)$, and a densely populated region with 6000 residents per square mile [13]. It is well documented that communities of color, those living in poverty, and in dense residential settings experience disparities in disease burden, morbidity, and mortality, for instance, HIV, diabetes, maternal coronary heart disease, all-cause mortality, breast cancer and hepatocellular carcinoma, alcohol/substance use, and of course COVID-19 to name just a few [7, 15-21].

Our objectives in this study were to estimate the true burden of SARS CoV-2 in the community, to determine the correlates of SARS CoV-2 prevalence and to determine if COVID-19 disparities seen by race/ethnicity were also reflected in SARS CoV-2 burden.

\section{Methods}

We utilized venue-based-sampling (VBS) to sample members of the community in Essex County. VBS is often employed as an alternative sampling method where simple random sampling is not feasible. In VBS, the venues are randomized as a proxy for randomizing the attendees of the venues. For example, VBS is the standard to sample certain populations at high risk for HIV infection where the population is known to attend identifiable venues. VBS is also the standard sampling method used by CDC for men who have sex with men in the USA in National
HIV Behavioral Surveillance (NHBS) and is used for other populations internationally but to our knowledge has not been used for SARS CoV-2 [22, 23]. North Jersey Community Research Initiative (NJCRI), which has over 20 years' experience in VBS as the implementing partner in NHBS for New Jersey, performed the VBS sampling for the present study by utilizing their mobile units, which have medical equipment, survey and phlebotomy space, and a private exam room. These units were also outfitted for COVID-19 by adding additional air filtration units and plexi-glass dividers to keep participants and staff safe. We identified venues using Google.com searches using the key words "Supermarkets in Essex County" and "Groceries in Essex County" to search for all potential locations. We identified 205 potential locations. Days and hours of operation were verified by contacting each venue directly. Venues, days, and times of operation were entered in an Excel spreadsheet. To map the venues, we used R 4.0.2 GUI (The R Foundation for Statistical Computing, Austria) to manipulate and analyze the data. Specifically, we used "tidygeocoder" package 1.0.1 to extract coordinates of each store from US Census and OpenStreetMap (OSM) dataset. For each month of sampling, approximately 42 venue-day-times were randomly sampled and scheduled for a sampling event. NJCRI's mobile units and staff used the VBS schedule and travelled to these grocery store sites. At the site, potential participants were systematically approached for a standard two-hour period of time, asked to stop for screening (intercept), screened for eligibility, and, if eligible, invited to participate and provide verbal informed consent. Participants who agreed to participate completed a short computer-based survey administered by study staff, using a tablet computer.

The survey included questions on basic self-reported demographics, COVID-19 symptoms (including: fever, dry cough, shortness of breath, chills, headache, sore throat, muscle pain/ aches, new loss of taste or smell, GI issues, neurologic symptoms) since January 2020, seeking viral PCR testing, PCR results, and seeking antibody testing and antibody results. Participants were then asked to provide a blood sample collected by finger stick with the Neoteryx Mitra Collection device to use for SARS-CoV-2 antibody testing [24]. Dried sticks were preserved by the NJCRI collection team or at the testing laboratory at room temperature for up to a week, according to manufacturer's instructions. At the time of testing, dried blood from the Mitra device was eluted overnight at $4{ }^{\circ} \mathrm{C}$, based on published protocols [24]. The eluate was diluted with $2 \%$ non-fat dry milk to1:80 and utilized for detection of IgG antibodies to recombinant receptor binding domain (RBD) of the SARS-CoV-2 Spike (100 ng/well), utilizing standard enzyme-linked immunosorbent assay (ELISA) protocols $[25,26]$. To interpret the ELISA data, we calculated a cut off of $\mathrm{OD}_{405}=0.3$ (mean +3 
standard deviations) by testing finger-stick eluates from 50 COVID-19 PCR-negative subjects.

Participants were given a result-return card to enable them to call in to the study team for their antibody results a week after sample receipt by the laboratory. Participants received \$25 in cash for their participation. This study had ethical approval from the Rutgers IRB. Survey data and lab data were merged in SAS (v9.4). Descriptive, bivariate, and multivariable analyses were conducted in SAS (v9.4).

\section{Results}

From September 15, 2020, to December 22, 2020, we conducted 92 randomly selected sampling events from geographically diverse locations in Essex County where we systematically approached patrons for screening. We approached 1349 individuals for screening. Of these, 928 (68.8\%) stopped and agreed to be screened, 928 (100\%) were eligible and agreed to participate, and 924 (99.6\%) consented and had complete survey and laboratory data for analysis.

Our sample population was mostly over 39 years of age $(n=593,64.2 \%)$ with a mean age of 44.7 years, mostly male at birth $(n=584,63.2 \%)$, predominantly Black $(n=656$, $71.0 \%$ ), and Latino ( $n=194,21.0 \%)$. A majority had a high school diploma or some college education $(n=652,70.5 \%)$. Only $343(37.1 \%)$ were employed full or part-time, while 398 (43.1\%) reported being unemployed. Personal annual income reported by participants was low, with almost half, $442(47.8 \%)$, reporting a personal annual income of less than $\$ 15,000$ while another 300 (32.5\%) reported an income of between $\$ 15,000$ and $\$ 35,000$. Homelessness was common in our sample, with 251 (27.2\%) reporting being homeless sometime in the 12 months preceding the survey and 181 (19.6\%) reporting being homeless at the time of the study. A majority of the sample reported living in Newark $(n=704$, $76.2 \%)$. Finally, $77.9 \%(n=720)$ of the sample reported having some sort of health insurance at the time of the study (Table 1).

In terms of SARS CoV-2 risks, just over half of the sample reported living alone (11.5\%) or with just one other person $(40.4 \%)$ and only $14.5 \%$ reported living in households with five or more persons. Only a small proportion of the sample $(5.0 \%)$ reported having had someone in their household diagnosed with COVID-19. The majority of the sample reported leaving their house daily $(84.2 \%)$, and only $4.8 \%$ reported only leaving home once a month or less than once a month. Only a few participants reported a previous positive PCR test result $(13 / 924,1.4 \%)$.

Only $6.5 \%$ of the sample reported any COVID-19 like symptoms since January 2020, while $45.9 \%$ reported having sought out a COVID-19 test. In total, 13 (1.4\%)
Table 1 Demographics, symptoms, testing seeking, and risk indicators and prevalence of SARS-CoV-2 antibody prevalence in a community sample, Essex County, NJ, 2020

\begin{tabular}{|c|c|c|}
\hline Variable & $\mathrm{N}(924)$ & $\%(100)$ \\
\hline \multicolumn{3}{|l|}{ Age } \\
\hline $18-21$ & 63 & 6.8 \\
\hline $22-29$ & 111 & 12.0 \\
\hline $30-39$ & 157 & 17.0 \\
\hline $40-49$ & 185 & 20.0 \\
\hline $50+$ & 408 & 44.2 \\
\hline \multicolumn{3}{|l|}{ Sex at birth } \\
\hline Female & 340 & 36.8 \\
\hline Male & 584 & 63.2 \\
\hline \multicolumn{3}{|l|}{ Race/ethnicity } \\
\hline Native American & 2 & 0.2 \\
\hline Asian & 6 & 0.6 \\
\hline Black & 656 & 71.0 \\
\hline White & 44 & 4.8 \\
\hline Latino & 194 & 21.0 \\
\hline Other & 11 & 1.2 \\
\hline Mixed & 6 & 0.6 \\
\hline Missing & 5 & 0.5 \\
\hline \multicolumn{3}{|l|}{ Educational attainment } \\
\hline None & 2 & 0.2 \\
\hline Grades $1-8$ & 29 & 3.1 \\
\hline Grade 9-11 & 170 & 18.4 \\
\hline HS diploma/GED & 466 & 50.4 \\
\hline Some college & 186 & 20.1 \\
\hline Bachelors & 53 & 6.4 \\
\hline Any grad school & 12 & 1.3 \\
\hline \multicolumn{3}{|l|}{ Employment status } \\
\hline Full time & 248 & 26.8 \\
\hline Part time & 95 & 10.3 \\
\hline Homemaker & 11 & 1.02 \\
\hline Student & 9 & 1.0 \\
\hline Retired & 42 & 4.6 \\
\hline Disabled & 120 & 13.0 \\
\hline Unemployed & 398 & 43.1 \\
\hline Missing & 1 & 0.1 \\
\hline \multicolumn{3}{|l|}{ Personal annual income } \\
\hline$\leq 14,999$ & 442 & 47.8 \\
\hline $15,000-35,000$ & 300 & 32.5 \\
\hline $36,000-50,000$ & 72 & 7.8 \\
\hline $51,001-80,000$ & 34 & 3.7 \\
\hline$>80,000$ & 76 & 8.2 \\
\hline Homeless 12 months prior to survey & 251 & 27.2 \\
\hline Homeless at time of survey & 181 & 19.6 \\
\hline \multicolumn{3}{|l|}{ Residential locality within Essex County } \\
\hline Belleville & 1 & 0.1 \\
\hline Bloomfield & 15 & 1.6 \\
\hline East Orange & 56 & 6.1 \\
\hline Elizabeth & 19 & 2.1 \\
\hline
\end{tabular}


Table 1 (continued)

\begin{tabular}{|c|c|c|}
\hline Variable & $\mathrm{N}(924)$ & $\%(100)$ \\
\hline Hillside & 2 & 0.2 \\
\hline Irvington & 59 & 6.4 \\
\hline Maplewood & 2 & 0.2 \\
\hline Montclair & 3 & 0.3 \\
\hline Newark & 704 & 76.2 \\
\hline Orange & 52 & 5.6 \\
\hline Roselle & 1 & 0.1 \\
\hline Short Hills & 1 & 0.1 \\
\hline Verona & 2 & 0.2 \\
\hline West Orange & 6 & 0.7 \\
\hline Homeless & 1 & 0.1 \\
\hline Has health insurance & 720 & 77.9 \\
\hline Any COVID-19 symptoms & 60 & 6.5 \\
\hline $\begin{array}{l}\text { Sought a polymerase chain reaction (PCR) virus } \\
\text { test }\end{array}$ & 424 & 45.9 \\
\hline Got PCR test & 407 & 44.1 \\
\hline Got PCR result & 383 & 41.5 \\
\hline \multicolumn{3}{|l|}{ Reported a positive previous PCR test } \\
\hline Yes & 13 & 1.4 \\
\hline No & 911 & 98.6 \\
\hline Sought Antibody (AB) test & 33 & 3.6 \\
\hline Got Ab test outside study & 29 & 3.1 \\
\hline Got $A b$ result outside study & 23 & 2.5 \\
\hline \multicolumn{3}{|l|}{ Reported a previous positive $\mathrm{AB}$ test } \\
\hline Yes & 5 & 0.5 \\
\hline No & 919 & 95.5 \\
\hline \multicolumn{3}{|l|}{ SARS CoV-2 Antibody result } \\
\hline Negative & 841 & 91.0 \\
\hline Positive & 83 & 9.0 \\
\hline \multicolumn{3}{|l|}{ Household size (including respondent) } \\
\hline 1 & 106 & 11.5 \\
\hline 2 & 373 & 40.4 \\
\hline 3 & 193 & 20.9 \\
\hline 4 & 118 & 12.8 \\
\hline $5+$ & 134 & 14.5 \\
\hline \multicolumn{3}{|l|}{ Any household COVID-19 diagnosis } \\
\hline Yes & 46 & 5.0 \\
\hline No & 878 & 95.0 \\
\hline \multicolumn{3}{|l|}{ How often respondent leaves the house each week } \\
\hline Everyday & 778 & 84.2 \\
\hline Weekly & 111 & 12.0 \\
\hline Once a month & 21 & 2.3 \\
\hline Less than once a month & 14 & 1.5 \\
\hline
\end{tabular}

participants reported receiving a positive SARS-CoV-2 PCR test result. While 33 participants (2.6\%) sought a SARS-CoV-2 antibody test outside of the study, only $0.5 \%$ of the sample reported a positive antibody result. Testing conducted in this study identified $83(9.0 \%)$ participants as positive for SARS-CoV-2 antibodies (Fig. 1).

In our bivariate analysis, only age $\left(X^{2}=16.1, \mathrm{p}<0.01\right)$, employment status $\left(X^{2} 4.8, \mathrm{p}=0.03\right)$, household diagnosis of COVID-19 $(p=0.01)$, and reporting a previous positive PCR test $(p<0.01)$ were significantly associated with testing positive for SARS CoV-2 antibodies. Antibody prevalence across age groups was not uniform nor was prevalence higher in older participants. Indeed, 18-21-year olds had the highest prevalence at $19.1 \%$. Interestingly, reporting homelessness in the 12 months preceding the survey was significantly negatively correlated with testing positive for SARS CoV-2 antibodies $\left(X^{2}=4.9, p=0.03\right)$ (Table 2).

In our multivariable analysis, we included age, race/ethnicity, sex assigned at birth, employment status, personal annual income, previous PCR test, household COVID-19 diagnosis, and homelessness in the 12 months prior to the study. While controlling for all of these variables simultaneously only age and household COVID-19 diagnosis were significantly associated with higher odds of testing positive for SARS CoV-2 antibodies. In terms of age, younger persons (18-21) had significantly higher odds (adjusted odds ratio $[\mathrm{AOR}]$ 5.6, 95\% confidence interval $[95 \% \mathrm{CI}] 1.8,17.2$, $p<0.01$ ) of testing positive for SARS CoV-2 antibodies compared to those aged 22-29. Those who previously had a positive PCR test had higher odds (AOR 11.3, 95\% CI 3.2, $38.9, p<0.01$ ) of testing positive for SARS CoV-2 antibodies. Notably, there were no significant differences in odds of testing positive for SARS CoV-2 antibodies in terms of race/ ethnicity where we compared Black and Latinx participants to other race participants.

\section{Discussion}

We successfully sampled a large number of residents of Essex County, New Jersey, for serological testing for SARS $\mathrm{CoV}-2$ antibodies. Our sample was predominantly Black and Latino and residing in Newark. Importantly, our findings suggest that the true burden of SARS-Cov-2 and the pool of persons potentially spreading the virus are slightly more than six times than that suggested by PCR testing. This burden from our robustly sampled community-based study is higher than most estimates obtained through studies of remnant blood samples from hospitals (4.2\% antibody prevalence), samples from staff at a public-school system (2.9\% antibody prevalence), and residents of a California county recruited with targeted Facebook ads (1.5\% antibody prevalence). These differences underscore the importance of conducting robust community-based sampling to determine the true burden of disease [27-29]. Moreover, with only $6.5 \%$ of the sample reporting any COVID-19-like symptoms, our finding suggests that the number of asymptomatic persons may be 


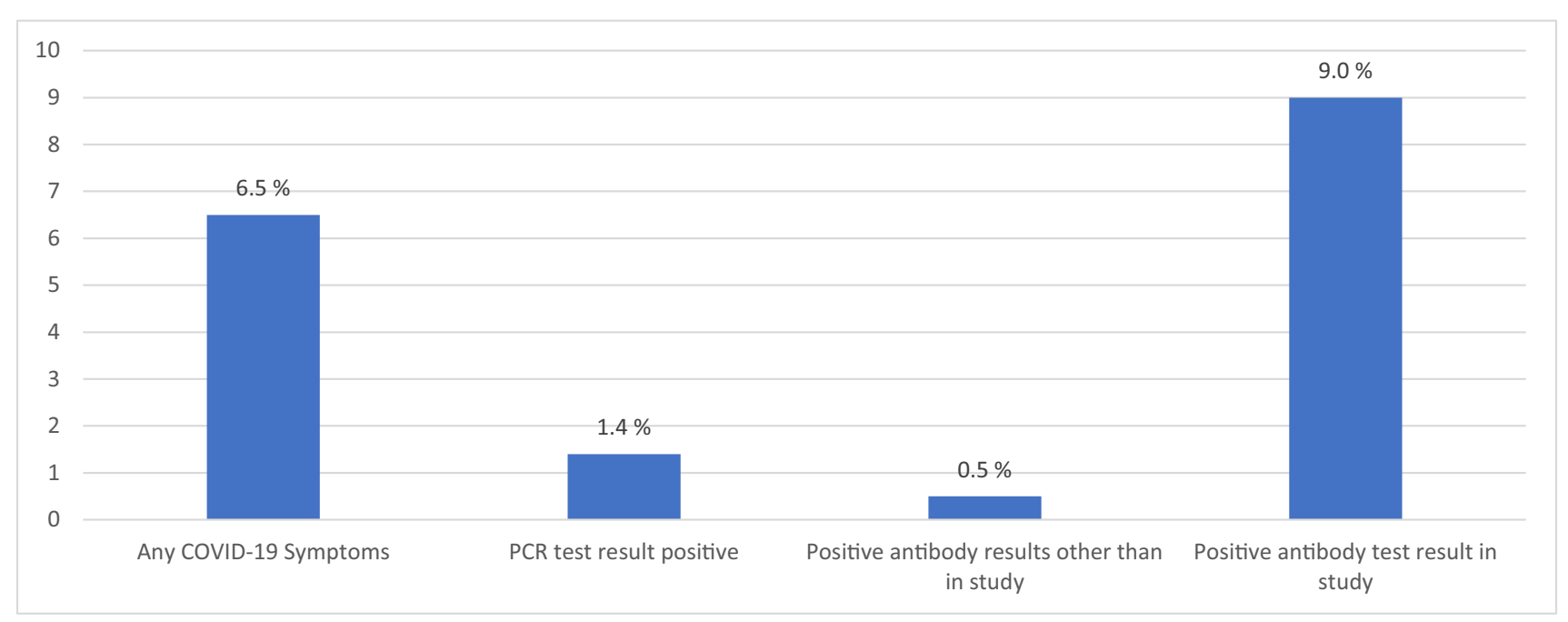

Fig. 1 COVID-19 and SARS-CoV-2 indicators among a community sample, Essex County, NJ, 2020

close to 1.5 times greater than anyone reporting symptoms (it is noted however that the symptoms listed might include other conditions).

In our bivariate analysis, we found that antibody prevalence was higher among younger and older persons, among those who were employed, those that were homeless sometime in the 12 months preceding the survey, and those who reported someone in their household being diagnosed with COVID-19. We did not find significant differences in SARS CoV-2 antibody prevalence by race/ethnicity, or frequency of leaving home. Other studies have also noted ongoing employment as potential risk factor serving as proxy for the risks experienced by front line workers. Indeed, our multivariable analysis suggests that only younger age and having previously received a positive PCR test for the virus were associated with SARS CoV-2 antibodies (Table 3).

Our study has limitations. VBS may not reach individuals who do not do their own shopping or live in settings where food is provided, thus introducing some form of sampling bias. Secondly, while Essex County is predominantly populated by Black/Latino individuals, our study oversampled these groups. This may be due to lower enthusiasm for participation among other race/ethnicity groups that may not be as altruistic or interested in the incentive provided by the study. Despite these limitations, we were able to sample a large number of individuals and estimate SARS-CoV-2 antibody prevalence in a county highly impacted by the current pandemic. Our findings underscore the importance of conducting seroprevalence studies in addition to case reporting to understand the true magnitude of SARS-CoV-2 impact on communities and plan for mitigation strategies to reduce transmission.

In conclusion, our study suggests that disparities in COVID-19 outcomes stem from potential upstream issues such as underlying conditions, access to testing, and access to care rather than disparities in exposure to the virus. In effect, these patterns manifest a biopsychosocial understanding of COVID-19, namely, the structural and social inequities that undermine health and interfere with access to effective healthcare by communities of color and other marginalized populations [9]. Our findings also suggest that other factors may be influencing these disparities. These factors may include hesitancy to get tested or hesitancy to seek care due to concerns about health care coverage/costs of care and/or fear and mistrust of the medical profession [8]. This bears further investigation particularly in relation to whether persons of color present for COIVD-19 care at more advance stages of the disease than other populations. SARS CoV-2 has exposed, in a dramatic way, the underlying systemic issues that influence health disparities in regard to race and ethnicity in the USA and point to ongoing vigilance in creating conditions that foster health equity both locally and internationally [8].

Author Contribution HFR, PW, RM, KDK, CR-D, and PNH designed, implemented, and contributed to this manuscript. HFR, PD, and CR-D supervised data collection and data management. MLG, PD, and RU were responsible for the lab aspects of the study. RM and HFR analyzed the data. All authors gave input to and approved the final manuscript. 
Table 2 Bivariate associations with SARS CoV-2 prevalence among a community sample, Essex County NJ, 2020

\begin{tabular}{|c|c|c|c|c|c|c|}
\hline \multirow[b]{2}{*}{ Variable } & \multicolumn{2}{|c|}{$\begin{array}{l}\text { SARS CoV-2 } \\
\text { negative (841) }\end{array}$} & \multicolumn{2}{|c|}{$\begin{array}{l}\text { SARS CoV-2 } \\
\text { positive (83) }\end{array}$} & \multirow[b]{2}{*}{$X^{2}$} & \multirow[b]{2}{*}{$p$} \\
\hline & $n$ & $\%$ & $n$ & $\%$ & & \\
\hline Age & & & & & 16.1 & $<0.01$ \\
\hline $18-21$ & 51 & 81.0 & 12 & 19.1 & & \\
\hline $22-29$ & 106 & 96.0 & 5 & 4.5 & & \\
\hline $30-39$ & 137 & 87.3 & 20 & 12.7 & & \\
\hline $40-49$ & 175 & 94.6 & 10 & 5.4 & & \\
\hline $50+$ & 372 & 91.2 & 36 & 12.1 & & \\
\hline Sex at birth & & & & & 1.1 & 0.3 \\
\hline Female & 305 & 89.7 & 35 & 10.3 & & \\
\hline Male & 536 & 91.8 & 48 & 8.2 & & \\
\hline Race/ethnicity & & & & & & $1.0^{*}$ \\
\hline Black & 596 & 90.9 & 60 & 9.2 & & \\
\hline Latinx & 177 & 91.2 & 17 & 8.8 & & \\
\hline Other & 68 & 91.9 & 6 & 8.1 & & \\
\hline Educational attainment & & & & & 3.4 & 0.2 \\
\hline Less than high school & 189 & 94.0 & 12 & 6.0 & & \\
\hline High school & 423 & 90.8 & 43 & 9.2 & & \\
\hline More than high school & 229 & 89.1 & 28 & 10.9 & & \\
\hline Employment status & & & & & 4.8 & 0.03 \\
\hline Employed & 303 & 88.3 & 40 & 11.7 & & \\
\hline Not working & 538 & 92.6 & 43 & 7.4 & & \\
\hline Personal annual income & & & & & 0.6 & 0.4 \\
\hline$<35,000$ & 678 & 91.4 & 64 & 8.6 & & \\
\hline$\geq 35,000$ & 163 & 89.6 & 19 & 10.4 & & \\
\hline Homeless in the 12 months prior to survey & & & & & 4.9 & 0.03 \\
\hline Yes & 237 & 94.4 & 14 & 10.4 & & \\
\hline No & 604 & 89.6 & 69 & 8.6 & & \\
\hline Homeless at time of survey & & & & & 2.3 & 0.1 \\
\hline Yes & 170 & 93.9 & 11 & 6.1 & & \\
\hline No & 671 & 90.3 & 72 & 9.7 & & \\
\hline Residential locality within Essex County & & & & & 0.4 & 0.8 \\
\hline Newark & 640 & 90.9 & 64 & 9.1 & & \\
\hline Other & 201 & 91.4 & 19 & 8.6 & & \\
\hline Household size (including respondent) & & & & & 7.3 & 0.1 \\
\hline 1 & 101 & 95.3 & 5 & 4.7 & & \\
\hline 2 & 945 & 92.5 & 28 & 7.5 & & \\
\hline 3 & 172 & 89.1 & 21 & 10.9 & & \\
\hline 4 & 102 & 86.4 & 16 & 13.6 & & \\
\hline $5+$ & 121 & 90.3 & 13 & 9.7 & & \\
\hline Any household COVID-19 diagnosis & & & & & & $0.01 *$ \\
\hline Yes & 37 & 80.4 & 9 & 19.6 & & \\
\hline No & 304 & 91.6 & 74 & 8.4 & & \\
\hline How often respondent leaves the house each week & & & & & 0.4 & 0.5 \\
\hline Everyday & 706 & 90.8 & 72 & 9.3 & & \\
\hline Less than everyday & 135 & 92.5 & 11 & 7.5 & & \\
\hline Reported a positive previous PCR test & & & & & & $<0.01^{*}$ \\
\hline Yes & 7 & 53.9 & 6 & 46.2 & & \\
\hline No & 834 & 91.6 & 77 & 8.5 & & \\
\hline
\end{tabular}

*Fisher exact test 
Table 3 Adjusted multivariable associations with SARS CoV-2 antibody prevalence among a community sample, Essex County NJ, 2020

\begin{tabular}{|c|c|c|c|}
\hline Variable & AOR & $95 \% \mathrm{CI}$ & $p$ \\
\hline \multicolumn{4}{|l|}{ Age } \\
\hline $18-21$ & 5.6 & $1.8,17.2$ & $<0.01$ \\
\hline $22-29$ & Ref & - & - \\
\hline $30-39$ & 3.7 & $1.3,10.5$ & 0.1 \\
\hline $40-49$ & 1.4 & $0.14,4.3$ & 0.6 \\
\hline $50+$ & 2.5 & $0.9,6.8$ & 0.07 \\
\hline \multicolumn{4}{|l|}{ Race/ethnicity } \\
\hline Black & 1.2 & $0.5,3.3$ & 0.7 \\
\hline Latinx & 1.1 & $0.4,3.1$ & 0.9 \\
\hline Other & Ref & - & - \\
\hline \multicolumn{4}{|l|}{ Birth sex } \\
\hline Female & 1.2 & $0.7,1.9$ & 0.5 \\
\hline Male & Ref & - & - \\
\hline \multicolumn{4}{|l|}{ Employment } \\
\hline Employed & 1.4 & $0.8,2.3$ & 0.2 \\
\hline Not working & Ref & - & - \\
\hline \multicolumn{4}{|c|}{ Personal annual income } \\
\hline$<35,000$ & 1.1 & $0.6,1.9$ & 0.8 \\
\hline$\geq 35,000$ & Ref & - & - \\
\hline \multicolumn{4}{|c|}{ Reported a positive previous PCR test } \\
\hline Yes & 11.3 & $3.2,38.9$ & $<0.01$ \\
\hline No & Ref & - & - \\
\hline \multicolumn{4}{|c|}{ Any household COVID-19 diagnosis } \\
\hline Yes & 2.1 & $0.9,4.9$ & 0.08 \\
\hline No & Ref & - & - \\
\hline \multicolumn{4}{|c|}{ Homeless in 12 months prior to the study } \\
\hline Yes & Ref & $0.9,3.2$ & 0.1 \\
\hline No & 1.7 & - & - \\
\hline
\end{tabular}

Funding This study was partially funded by Pfizer \# 61618273 and the Turell Foundation.

Data Availability By request to the first author.

Code Availability Not applicable.

\section{Declarations}

Disclaimer Neither funder had a role in the design, implementation, or analysis of this study.

Ethics Approval Ethics approval was given by the Rutgers University IRB.

Consent to Participate All participants provided verbal informed consent.

Conflict of Interest The authors declare no competing interests.

\section{References}

1. Zelner J, Trangucci R, Naraharisetti R, Cao A, Malosh R, Broen $\mathrm{K}$, et al. Racial disparities in coronavirus disease 2019 (COVID19) mortality are driven by unequal infection risks. Clin Infect Dis. 2021;72(5):e88-95.

2. Aleligne YK, Appiah D, Ebong IA. Racial disparities in coronavirus disease 2019 (covid-19) outcomes. Curr Opin Cardiol. 2021. https://doi.org/10.1097/HCO.0000000000000847.

3. Anderson-Carpenter KD, Neal ZP. Racial disparities in COVID19 impacts in Michigan, USA. J Racial Ethn Health Disparities. 2021.

4. Pierce JB, Harrington K, McCabe ME, Petito LC, Kershaw KN, Pool LR, et al. Racial/ethnic minority and neighborhood disadvantage leads to disproportionate mortality burden and years of potential life lost due to COVID-19 in Chicago, Illinois. Health Place. 2021;68:102540.

5. Dai CL, Kornilov SA, Roper RT, Cohen-Cline H, Jade K, Smith B, et al. Characteristics and factors associated with COVID-19 infection, hospitalization, and mortality across race and ethnicity. Clin Infect Dis. 2021.

6. Tai DBG, Shah A, Doubeni CA, Sia IG, Wieland ML. The disproportionate impact of COVID-19 on racial and ethnic minorities in the United States. Clin Infect Dis. 2021;72(4):703-6.

7. Weiser JK, Tie Y, Beer L, Neblett Fanfair R, Shouse RL. Racial/ ethnic and income disparities in the prevalence of comorbidities that are associated with risk for severe COVID-19 among adults receiving HIV care, United States, 2014-2019. J Acquir Immune Defic Syndr. 2021;86(3):297-304.

8. Halkitis PN. A new public health psychology to mend the chasm between public health and clinical care. Am Psychol. 2020;75(9):1289-96.

9. Halkitis PN. Managing the COVID-19 pandemic: biopsychosocial lessons gleaned from the AIDS epidemic. J Public Health Manag Pract. 2021;27 Suppl 1, COVID-19 and public health: looking back, moving forward:S39-S42.

10. Shiau S, Krause KD, Valera P, Swaminathan S, Halkitis PN. The burden of COVID-19 in people living with HIV: a syndemic perspective. AIDS Behav. 2020;24(8):2244-9.

11. Oran DP, Topol EJ. The proportion of SARS-CoV-2 infections that are asymptomatic: a systematic review. Ann Intern Med. 2021.

12. Department of Health NJ. New Jersey COVID-19 Information Hub 2021 [Available from: https://covid19.nj.gov/forms/datad ashboard. Accessed 28 Sept 2021.

13. Census US. American Fact Finder 2018 [Available from: https:// factfinder.census.gov/faces/nav/jsf/pages/index.xhtml. Accessed 28 Sept 2021.

14. WHO. WHO Emergency Dashboard: WHO; 2021 [Available from: https://covid19.nj.gov/index.html\#live-updates. Accessed 28 Sept 2021

15. Arayasirikul S, Pomart WA, Raymond HF, Wilson EC. Unevenness in health at the intersection of gender and sexuality: sexual minority disparities in alcohol and drug use among transwomen in the San Francisco Bay Area. J Homosex. 2018;65(1):66-79.

16. Beer L, Bradley H, Mattson CL, Johnson CH, Hoots B, Shouse $\mathrm{RL}$, et al. Trends in racial and ethnic disparities in antiretroviral therapy prescription and viral suppression in the United States, 2009-2013. J Acquir Immune Defic Syndr. 2016;73(4):446-53.

17. Beer L, Oster AM, Mattson CL, Skarbinski J, Project MM. Disparities in HIV transmission risk among HIV-infected black and white men who have sex with men, United States, 2009. AIDS. 2014;28(1):105-14.

18. Reback CJ, Clark K, Holloway IW, Fletcher JB. Health disparities, risk behaviors and healthcare utilization among transgender 
women in Los Angeles County: a comparison from 1998-1999 to 2015-2016. AIDS Behav. 2018;22(8):2524-33.

19. Watt GP, John EM, Bandera EV, Malone KE, Lynch CF, Palmer $\mathrm{JR}$, et al. Race, ethnicity and risk of second primary contralateral breast cancer in the United States. Int J Cancer. 2021.

20. Malek AM, Wilson DA, Turan TN, Mateus J, Lackland DT, Hunt KJ. Maternal coronary heart disease, stroke, and mortality within 1,3 , and 5 years of delivery among women with hypertensive disorders of pregnancy and pre-pregnancy hypertension. J Am Heart Assoc. 2021;10(5):e018155.

21. Wong RJ, Saab S, Konyn P, Sundaram V, Khalili M. Ruralurban geographical disparities in hepatocellular carcinoma incidence among US adults, 2004-2017. Am J Gastroenterol. 2021;116(2):401-6.

22. Wang J, Li D, Wiltse A, Emo J, Hilchey SP, Zand MS. Application of volumetric absorptive microsampling (VAMS) to measure multidimensional anti-influenza IgG antibodies by the mPlex-Flu assay. J Clin Transl Sci. 2019;3(6):332-43.

23. Davidow A, Kanaujia GV, Shi L, Kaviar J, Guo X, Sung N, et al. Antibody profiles characteristic of Mycobacterium tuberculosis infection state. Infect Immun. 2005;73(10):6846-51.

24. Gennaro ML, Affouf M, Kanaujia GV, Brusasca PN, Mangura B, Reichman L. Antibody markers of incident tuberculosis among HIV-infected adults in the USA: a historical prospective study. Int J Tuberc Lung Dis. 2007;11(6):624-31.
25. Routledge I, Epstein A, Takahashi S, Hakim J, Janson O, Duarte $\mathrm{E}$, et al. Citywide serosurveillance of the initial SARS-CoV-2 outbreak in San Francisco. Res Sq. 2021.

26. Lopez L, Nguyen T, Weber G, Kleimola K, Bereda M, Liu Y, et al. Seroprevalence of anti-SARS-CoV-2 IgG antibodies in the staff of a public school system in the Midwestern United States. medRxiv. 2020.

27. Bendavid E, Mulaney B, Sood N, Shah S, Ling E, Bromley-Dulfano R, et al. COVID-19 Antibody seroprevalence in Santa Clara County, California. 2020:2020.04.14.20062463.

28. Martino RJ, Krause KD, Griffin M, LoSchiavo CE, Comer-Carruthers C, Karr A, et al. A national survey of COVID-19 testing in the US. LGBTQ Populations. Public Health Rep. 2021;IN PRESS.

29. Weaver KE, Rowland JH, Bellizzi KM, Aziz NM. Forgoing medical care because of cost: assessing disparities in healthcare access among cancer survivors living in the United States. Cancer. 2010;116(14):3493-504.

Publisher's Note Springer Nature remains neutral with regard to jurisdictional claims in published maps and institutional affiliations. 